\title{
Towards the first measurement of matter-antimatter gravitational interaction
}

C. Evans ${ }^{1,2, a}$, S. Aghion ${ }^{1,2}$, C. Amsler ${ }^{3}$, G. Bonomi ${ }^{4,5}$, R. S. Brusa ${ }^{6,7}$, M. Caccia $^{2,8}$, R. Caravita9,10, F. Castelli ${ }^{2,11}$, G. Cerchiari ${ }^{12}$, D. Comparat ${ }^{13}$, G. Consolati ${ }^{2,14}$, A. Demetrio ${ }^{15}$, L. Di Noto ${ }^{9,10}$, M. Doser $^{16}$, M. Fani ${ }^{9,10,16}$, R. Ferragut ${ }^{1,2}$, J. Fesel ${ }^{16}$, A. Fontana $^{5}$, S. Gerber ${ }^{16}$, M. Giammarchi ${ }^{2}$, A. Gligorova $^{3}$, F. Guatieri6,7, S. Haider ${ }^{16}$, A. Hinterberger ${ }^{16}$, H. Holmestad ${ }^{17}$, A. Kellerbauer ${ }^{12}$, O. $K_{\text {Khalidova }}{ }^{15}$, D. Krasnický ${ }^{9,10}$, V. Lagomarsino ${ }^{9,10}$, P. Lansonneur ${ }^{18}$, P. Lebrun ${ }^{18}$, C. Malbrunot ${ }^{3,16}$, S. Mariazzi ${ }^{3}$, J. Marton ${ }^{3}$, V. Matveev ${ }^{19,20}$, Z. Mazzotta ${ }^{2,11}$, S. R. Müller ${ }^{15}$, G. Nebbia ${ }^{21}$, P. Nedelec $^{18}$, M. Oberthaler ${ }^{15}$, N. Pacifico ${ }^{16,23}$, D. Pagano ${ }^{4,5}$, L. Penasa ${ }^{6,7}$, V. Petracek ${ }^{24}$, F. Prelz ${ }^{2}$, M. Prevedelli ${ }^{25}$, L. Ravelli, ${ }^{6,7}$, B. Rienaecker ${ }^{16}$, J. Robert ${ }^{13}$, O. M. Røhne ${ }^{22}$, A. Rotondi ${ }^{5,26}, \mathrm{H}$. Sandaker $^{17}$, R. Santoro ${ }^{2,8}$, L. Smestad ${ }^{16,27}$, F. Sorrentino ${ }^{9,10}$, G. Testera ${ }^{10}$, I. C. Tietje ${ }^{16}$, E. Widmann $^{3}$, P. Yzombard ${ }^{12}$, C. Zimmer ${ }^{12,16,22}$, J. Zmeskal ${ }^{3}$, and N. Zurlo ${ }^{5,28}$

${ }^{1}$ LNESS, Department of Physics, Politecnico di Milano, via Anzani 42, 22100, Como, Italy

${ }^{2}$ INFN Milano, via Celoria 16, 20133, Milano, Italy

${ }^{3}$ Stefan Meyer Institute for Subatomic Physics, Austrian Academy of Sciences, Boltzmanngasse 3, 1090 Vienna, Austria

${ }^{4}$ Department of Mechanical and Industrial Engineering, University of Brescia, via Branze 38, 25123 Brescia, Italy

${ }^{5}$ INFN Pavia, via Bassi 6, 27100 Pavia, Italy

${ }^{6}$ Department of Physics, University of Trento, via Sommarive 14, 38123 Povo, Trento, Italy

${ }^{7}$ TIFPA/INFN Trento, via Sommarive 14, 38123 Povo, Trento, Italy

${ }^{8}$ Department of Science, University of Insubria, Via Valleggio 11, 22100 Como, Italy

${ }^{9}$ Department of Physics, University of Genova, via Dodecaneso 33, 16146 Genova, Italy

${ }^{10}$ INFN Genova, via Dodecaneso 33, 16146 Genova, Italy

${ }^{11}$ Department of Physics, University of Milano, via Celoria 16, 20133 Milano, Italy

${ }^{12}$ Max Planck Institute for Nuclear Physics, Saupfercheckweg 1, 69117 Heidelberg, Germany

${ }^{13}$ Laboratoire Aimé Cotton, Université Paris-Sud, ENS Paris Saclay, CNRS, Université Paris-Saclay, 91405 Orsay Cedex, France

${ }^{14}$ Department of Aerospace Science and Technology, Politecnico di Milano, via La Masa 34, 20156, Milano, Italy

${ }^{15}$ Kirchhoff-Institute for Physics, Heidelberg University, Im Neuenheimer Feld 227, 69120 Heidelberg, Germany

${ }^{16}$ Physics Department, CERN, 1211 Geneva 23, Switzerland

${ }^{17}$ Department of Physics, University of Oslo, Sem Slandsvei 24, 0371 Oslo, Norway

${ }^{18}$ Institute of Nuclear Physics, CNRS/IN2p3, University of Lyon 1, 69622 Villeurbanne, France

${ }^{19}$ Institute for Nuclear Research of the Russian Academy of Science, Moscow 117312, Russia

${ }^{20}$ Joint Institute for Nuclear Research, 141980 Dubna, Russia

${ }^{21}$ INFN Padova, via Marzolo 8, 35131 Padova, Italy

${ }_{22}$ Department of Physics, Heidelberg University, Im Neuenheimer Feld 226, 69120 Heidelberg, Germany

${ }^{23}$ Institute of Physics and Technology, University of Bergen, Allégaten 55, 5007 Bergen, Norway

ae-mail: craig.evans@polimi.it 


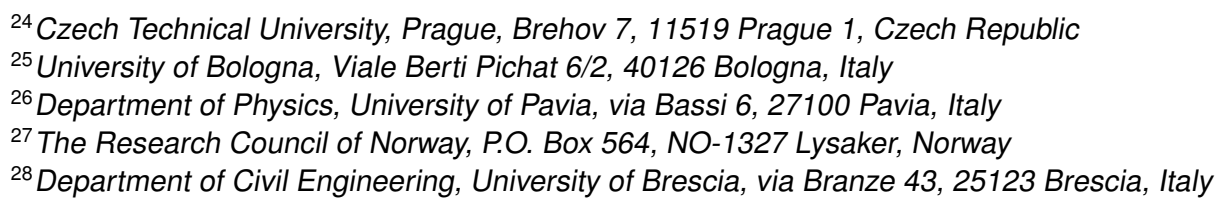

\begin{abstract}
The AEgIS (Antimatter Experiment: Gravity, Interferometry, Spectroscopy) is a CERN based experiment with the central aim to measure directly the gravitational acceleration of antihydrogen. Antihydrogen atoms will be produced via charge exchange reactions which will consist of Rydberg-excited positronium atoms sent to cooled antiprotons within an electromagnetic trap. The resulting Rydberg antihydrogen atoms will then be horizontally accelerated by an electric field gradient (Stark effect), they will then pass through a moiré deflectometer. The vertical deflection caused by the Earth's gravitational field will test for the first time the Weak Equivalence Principle for antimatter. Detection will be undertaken via a position sensitive detector. Around $10^{3}$ antihydrogen atoms are needed for the gravitational measurement to be completed. The present status, current achievements and results will be presented, with special attention toward the laser excitation of positronium (Ps) to the $\mathrm{n}=3$ state and the production of Ps atoms in the transmission geometry.
\end{abstract}

\title{
1 Introduction
}

The observable universe appears to consist of practically only matter with an unexplained absence of antimatter. The reason for this is still unknown. Experiments based at CERN's Antiproton Decelerator (AD) [1] are attempting to shed more light on this puzzle. These experiments aim to find asymmetrical properties between hydrogen and antihydrogen $(\bar{H})$, its antimatter counterpart. The symmetry between particle and antiparticle is described by the CPT (Charge conjugation, Parity, Time reversal) theorem [2]. The main properties described by the CPT theorem is that both particles and antiparticles have the same mass, momentum, energy and spin, whilst the additive quantum numbers (charge, baryon number, etc.) are inverted. Another subject of investigation is gravity. General relativity considers gravity as a geometric property of space and time and has not yet successfully included in any Quantum Field Theory (QFT), and thus the CPT theorem does not apply. The experimental study of the Weak Equivalence Principle (WEP) for antimatter could lead to a possible quantum theory of gravity [3]. WEP dictates that the effect of a gravitational field on a system does not depend on its composition or structure [4]. WEP has been tested to a precision of $1.8 \times 10^{-13}$ with ordinary matter [5].

Electrically charged antiparticles (e.g. positrons and antiprotons) have been trapped within a combination of magnetic and electric fields, in the form of Penning traps [6]. Within such trap-based systems, residual stray electric and magnetic fields affect these antiparticles [7] and render any such gravitational observation impossible. In 2002, the ATHENA experiments managed to create lowenergy antihydrogen $(\bar{H})$ [8]. This neutral antiatom was formed via a three-body reaction by mixing trapped antiprotons $(\bar{p})$ with positrons $\left(e^{+}\right)$at low energies. This opened the possibility to test WEP on antimatter $\bar{H}$ because it is not affected by the stray fields mentioned before. Since then, the ALPHA collaboration performed a preliminary measurement of the Earth's gravitational effect on $\bar{H}$, trapped magnetically. As a result, the gravitational acceleration of $\bar{H}$ was constrained to within $\approx 100$ times the $g$ value for matter [9].

The AEgIS collaboration, proposed in $2007[10,11]$ plans to measure the effect of gravitational field on $\bar{H}$ atoms to $1 \%$ accuracy using a cold beam of $\bar{H}$ (the concept is shown in Fig. 1). $\bar{H}^{*}$ will be 


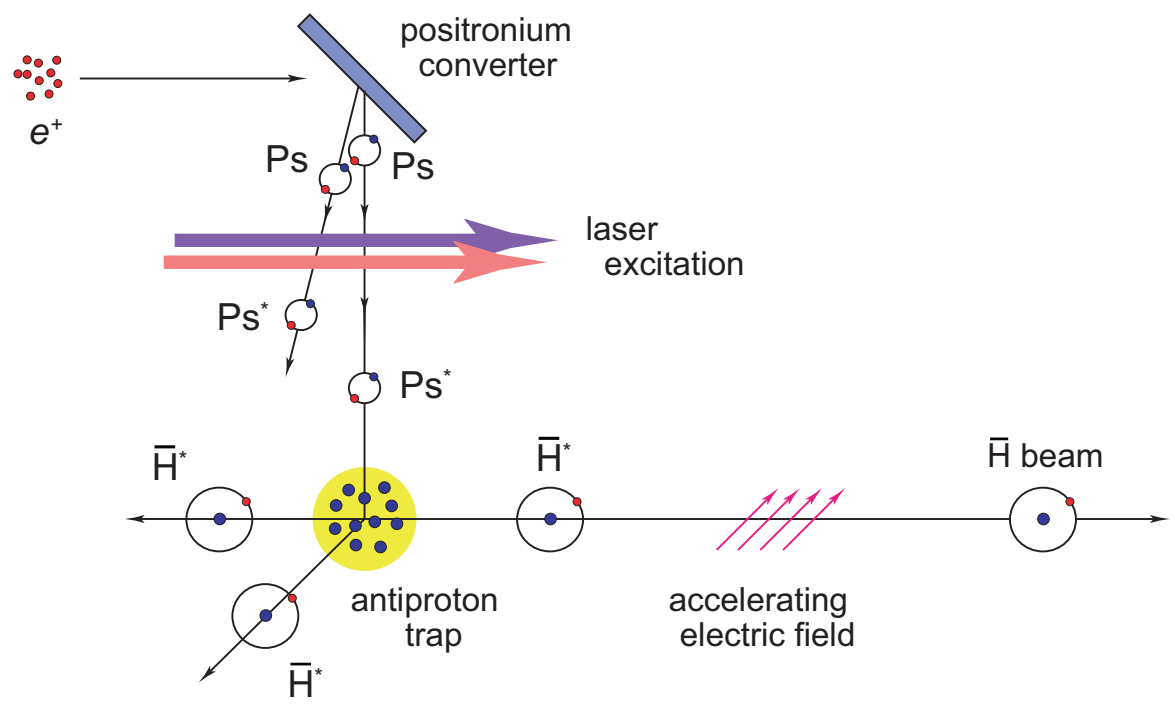

Figure 1. Antihydrogen formation process using the charge exchange reaction between Ps and cold $\bar{p}$.

formed in a novel way. Introducing cold trapped $\bar{p}$ to cold, Rydberg-state positronium $\left(P s^{*}\right)$. $P s^{*}$ is the bound state of an electron $e^{-}$and a $e^{+}$[12] laser excited to Rydberg levels to increase the principle quantum number $\mathrm{n}$ and its lifetime. The charge exchange reaction [13] described as

$$
P s^{*}+\bar{p} \rightarrow \bar{H}^{*}+e^{-},
$$

where the Rydberg antihydrogen $\left(\bar{H}^{*}\right)$ [14] temperature after formation is predicted to be close to the initial trapped antiproton temperature [15]. The great benefit of $\bar{H}^{*}$ is that the electric dipole moment is large, allowing Stark acceleration [16] to occur. This in turn will allow the realisation of a pulsed beam. The $\bar{H}^{*}$ is predicted to decay to the ground state during flight prior to the gravity measurement region. Excitation of Ps to Rydberg states also directly benefits the charge exchange process since the cross section is depends on the fourth power of the Ps principal quantum number [14].

\section{The AEgIS Experimental Apparatus}

The AEgIS apparatus, shown in Fig. 2, implements superconducting magnets, a $5 \mathrm{~T}$ and a $1 \mathrm{~T}$ magnet which surround the initial antiproton trapping and the $\bar{H}$ formation regions, respectively. A series of cylindrical electrodes inside each magnet form a Penning-Malmberg trap arrangement [6]. This allows radial and axial confinement of the charged particles. A bunch of $3 \times 10^{7} \bar{p}$ with kinetic energies around $5 \mathrm{MeV}$ initially are delivered from the $\mathrm{AD}$. Before entering the main apparatus, a set of aluminium foils (degraders) reduce the incoming $\bar{p}$ energy to a few $\mathrm{keV}$. A fraction of these decelerated $\bar{p}$ are trapped within the $5 \mathrm{~T}$ region high voltage electrodes. After the initial trapping, the antiprotons are electron cooled [17] by interactions with an $e^{-}$cloud previously stored inside the trap. The $\bar{p}$ are cooled to a few kelvin before the $e^{-}$cloud is removed by a voltage kick. The $\bar{p}$ are then transferred to the $1 \mathrm{~T}$ region.

To obtain Ps, the experiment initially utilizes a positron pulsed beam, shown in Fig. 3. The source of positrons originates from the decay of a ${ }^{22} \mathrm{Na}$ source $(50 \mathrm{mCi})$. The positrons are then moderated 


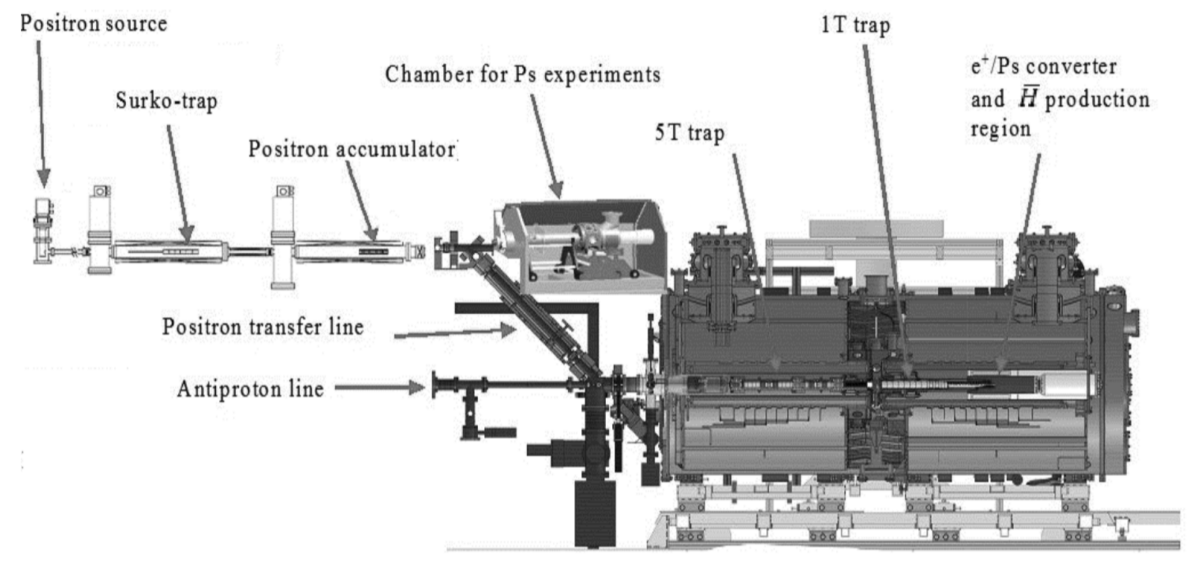

Figure 2. The experimental setup scheme of the AEgIS experiment. The positron beam and test chamber on the upper left area are presented in more detail in Fig. 3. This image excludes the moiré deflectometer (presented later in Fig 4 and Fig 5).

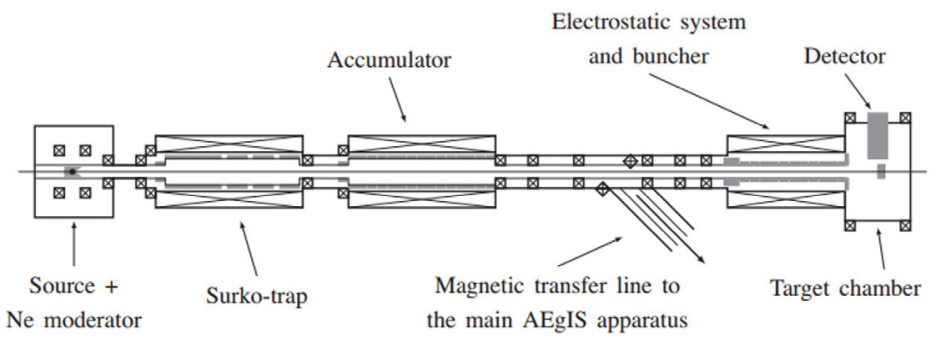

Figure 3. Side view of the positron accumulation setup, including the Ps test chamber at the far right. (Image from Ref. [21]).

by a solid neon layer, created by freezing $\mathrm{Ne}$ gas onto the source and surrounding walls at around $7 \mathrm{~K}$. These moderated positrons are then trapped in a Surko trap, which, like the antiproton section, employs an arrangement of electrodes and cylindrical magnets to confine them in both radial and axial directions [18]. The accumulated positron bunch is then directed towards a mesoporous $e^{+} / \mathrm{Ps}$ conversion target [19] $\left(\approx 10^{8}\right.$ positrons per bunch). The positrons enter the material, then form Ps inside the mesopores. The newly formed Ps atoms thermalize via collisions and finds their way out of the pores into vacuum [20], in the direction towards the trapped low energy antiproton plasma inside the $1 \mathrm{~T}$ region.

A Ps test chamber, located at the end of the positron system (Fig. 2 and Fig. 3), is used for Ps formation and excitation tests for separate and supplementary experimentation (for the main experiment) [21]. The positrons pass a magnetic field terminator in a bunch of $7 \mathrm{~ns}$ FWHM (full width half maximum) which is then focused down to a $4 \mathrm{~mm}$ diameter spot, onto the target. Two glass fibres from the laser section are also installed to allow laser excitation of the Ps near the target. For use with Ps, a selection of lasers are implemented to allow a first step excitation, from ground to an the $n=3$ 
state with a UV laser then to a Rydberg state using an IR laser [22]. The Rydberg-excited Ps atoms would then interact with the $\bar{p}$ to allow $\bar{H}$ formation via charge exchange.

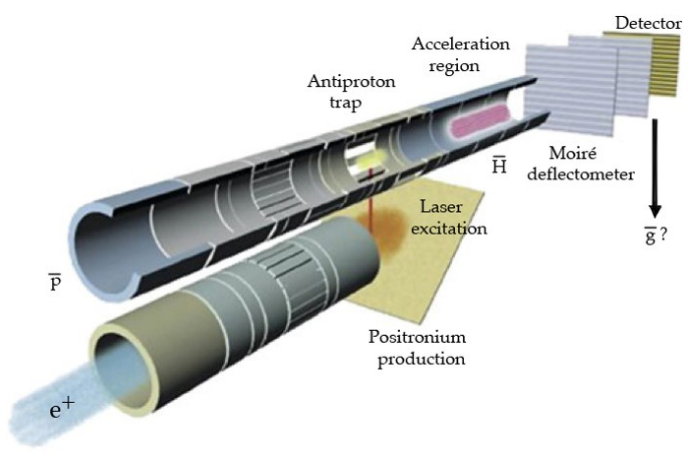

Figure 4. 3D view of the antihydrogen formation region (not to scale).

For the gravity measurement, the beam will pass through a classical moiré deflectometer featuring two gratings and a position sensitive detector [23], as shown schematically in Fig. 4. The deflectometer fringes as subject to a vertical shift $h$ would be caused by the gravitational effect on the incoming pulses of $\bar{H}$, described as

$$
h=g_{h}\left(\frac{L}{v}\right)^{2},
$$

where $L$ is the distance between the two gratings, $g_{h}$ is the gravitational acceleration experienced on $\bar{H}$, and $v$ is the perpendicular velocity of the $\bar{H}$ after the Stark acceleration before the moiré deflectometer. Different velocities $v$ will be tested to extract the $g_{h}$ value [24]. A more detailed sketch of the deflectometer is shown in Fig. 5. A small-scale version of the deflectometer has been tested with antiprotons [25].

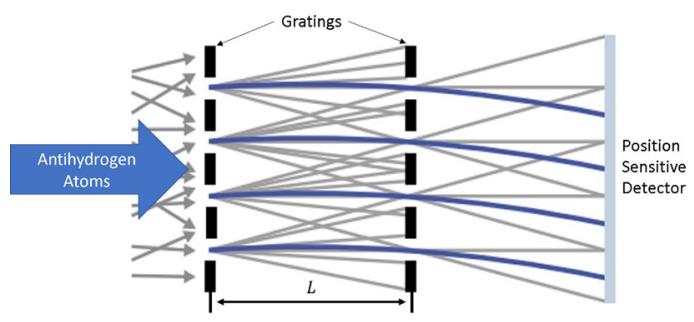

Figure 5. Sketch of the moiré deflectometer showing the antiprotons passing through the two gratings then hitting the position sensitive detector. The dark blue line indicates the expected/predicted downward shift caused by the gravitational force of the earth. In the measurement of Ref. [25], the position sensitive detector was in the form of a photographic emulsion [26]. 

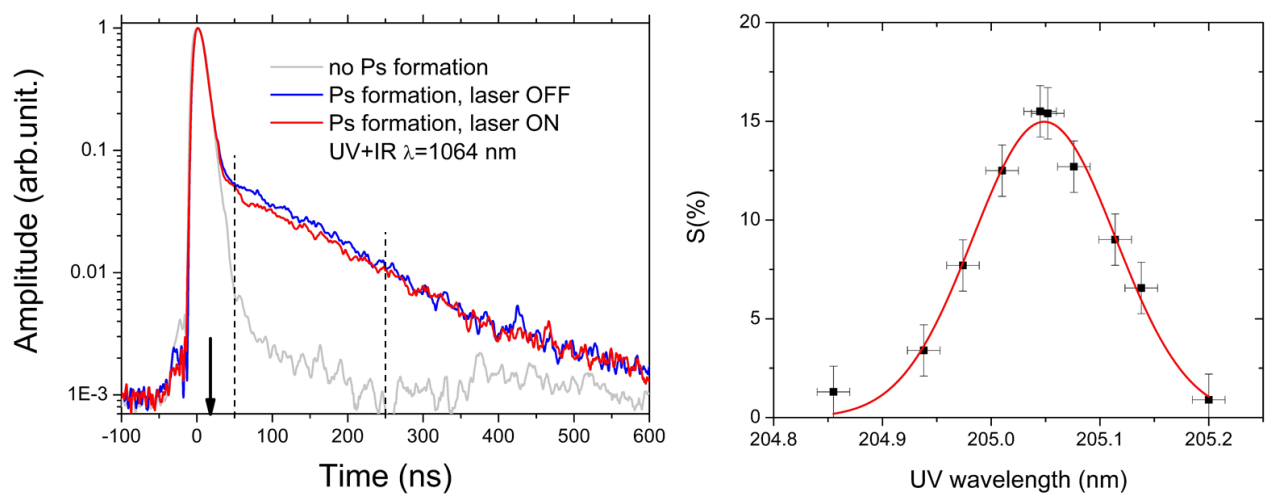

Figure 6. SSPALS measurement (left) containing 3 lifetime spectra. Light gray (background), Ps emitted into vacuum with laser OFF (blue) and with the UV+IR lasers ON $(205.05+1064 \mathrm{~nm})($ red). The 50 to $250 \mathrm{~ns}$ region indicated by the vertical dashed lines was used to determine the $S$ parameter (see Eq. (3)) for $n=3$ (right). This graph illustrates the Ps excitation efficiency as a function of the UV laser wavelength (both images from Ref. [28]).

\section{Recent Experimental outcomes}

\subsection{Positronium production in reflection and excitation}

Positronium (Ps) can be formed in two states: Para-Positronium p-Ps (singlet state) and orthopositronium o-Ps (triplet state). In vacuum, both states annihilate into pure energy in the form of gamma rays, but they differ in lifetime and the quantity of gamma rays produced. p-Ps annihilates mainly into two gamma rays with a mean lifetime of $125 \mathrm{ps}$ and o-Ps mainly into three gamma rays at a mean lifetime of $142 \mathrm{~ns}$. o-Ps has a long enough lifetime to allow an increase in its life prior to annihilation via laser excitation [27].

AEgIS has recently performed, for the first time, Ps laser excitation to the $n=3$ state. This was done with the use of a laser pulse of wavelength around $205 \mathrm{~nm}$ to first reach the $\mathrm{n}=3$ state, then a second $1064 \mathrm{~nm}$ laser pulse for photoionization [28]. This experiment was performed in the Ps test chamber using a reflective target. $20 \mathrm{~ns}$ bunches of positrons (each with $3 \times 10^{7}$ ) were accelerated and focused onto the target at a kinetic energy of $3.3 \mathrm{keV}$. Ps is formed inside the target and o-Ps is then emitted into vacuum. The o-Ps in vacuum was detected via a $20 \times 25 \times 25 \mathrm{~mm}^{3} \mathrm{PbWO}_{4}$ scintillator coupled with a Hamamatsu R11265-100 PMT (Photo Multiplier Tube). With this setup, the single shot positron annihilation lifetime spectroscopy (SSPALS) method [29] was used to determine the Ps lifetime spectra via the detection of the annihilating gamma rays, as shown in Fig. 6.

The region between the two vertical dotted lines in the lifetime spectra shows a difference between laser on and laser off states. The decrease inside this region indicates an extension of the o-Ps lifetime, caused by the laser excitation. From this change it was possible to determine the efficiency of the emitted o-Ps excited via the $\mathrm{S}$ parameter defined as,

$$
S=\left(f_{o f f}-f_{o n}\right) / f_{o f f},
$$

where $f_{\text {on }}$ and $f_{\text {off }}$ are the areas within the selected section of the spectra (dotted line), shown in the left image of Fig. 6, when the laser is turned on and off respectively. A scan around this wavelength was also performed (as shown in the right pane of Fig. 6) and it was found that the dependence of 
the $\mathrm{S}$ parameter on the wavelength follows a Gaussian shape (caused by the Ps thermal distribution). The combined excitation and photoionization efficiency was determined to be around $15 \%$ [28]. The maximum corresponds to a wavelength of $205.05 \pm 0.02 \mathrm{~nm}$. This is in agreement with the prevision of a previous work [22].

\subsection{Positronium production in transmission}

For the formation of antihydrogen via charge exchange, cold antiprotons and an efficient source of Ps are needed. Currently, a $e^{+} /$Ps reflective convertor is implemented as the source of Ps [19]. A proposed upgrade to the system is the development and implementation of a Ps transmission target to increase the quantity of themalized Ps interacting with the trapped antiprotons and to simplify the overall apparatus. This involves moving from the current off-axis to a more on-axis linear design. A $e^{+} /$Ps converter was characterised within the Ps test chamber as a possible alternative to the current reflective geometry target [30].

This target consists of an ultra-porous layer of meso-structured silica (thickness around $750 \mathrm{~nm}$ ). This coating was evaporated (via e-gun) in a glancing angle deposition set-up [31] onto a $20 \mathrm{~nm}$ carbon foil (to provide a platform and mechanical stability for the film). The target was placed onto a movable actuator within the central region of the chamber to allow the converter to face upstream and then downstream for reflection and transmission measurements respectively. $\mathrm{A} \mathrm{PbWO}_{4}$ scintillator (located externally) was used to determine the Ps yield from the SSPALS spectra. The Ps yield in vacuum was found to be around $7 \%$ for both geometries, similar to previously reported experimental results $[27,31]$. In the both the reflection and transmission orientations, from the SSPALS spectra (shown in the right pane Fig. 7) are observed to produce the same output of o-Ps. This in turn opens the possibility for using these targets in both orientations.
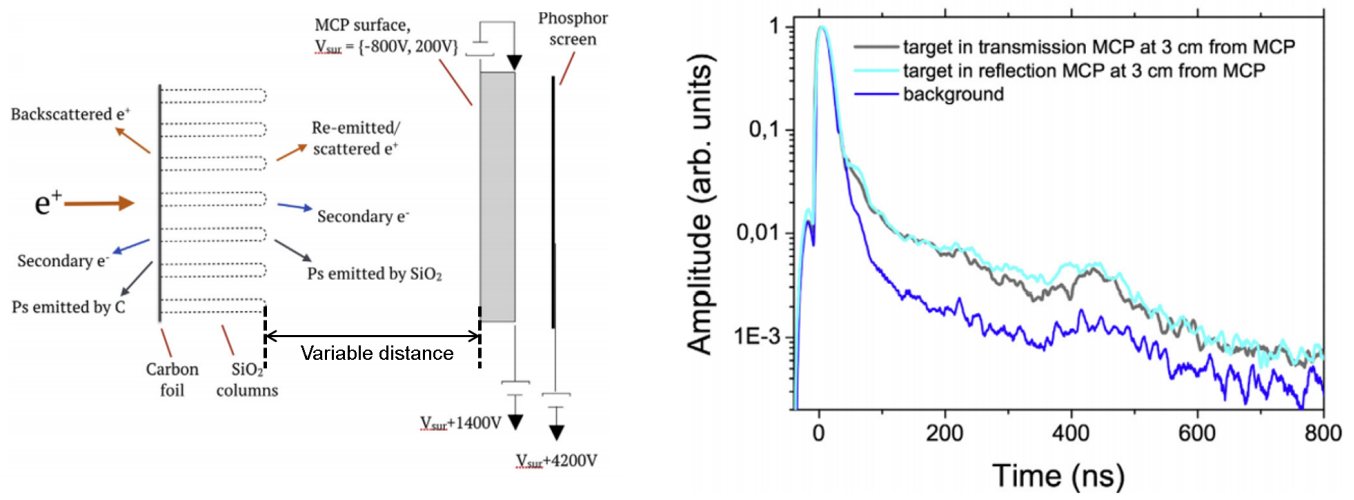

Figure 7. Sketch of the transmission measurement configuration (left) where the MCP is placed in front of the target to detect the secondary charged species produced. The target was inverted for reflection measurement. The lifetime spectra (right) are produced via SSPALS measured in reflection and transmission at an MCP-target distance of $3 \mathrm{~cm}$ (both images from Ref. [30]).

An imaging MCP was implemented to monitor the quantity of charged species (electrons and positrons) emitted from the target in reflection and transmission. The images presented in Fig. 8 were taken in transmission geometry for different potentials (between $-800 \mathrm{~V}$ and $+200 \mathrm{~V}$ ), applied onto the frontal face of the MCP (see right panee of Fig. 7). From these MCP images, it was determined 
that around $10 \%$ of positrons with energies around $1.2 \mathrm{keV}$ were re-emitted (from the initial positron implantation energy of $3.3 \mathrm{keV}$ ) [30]. Secondary electron emission in transmission was measured to be around $17 \%$ from the initial positron bunch in the forward direction.

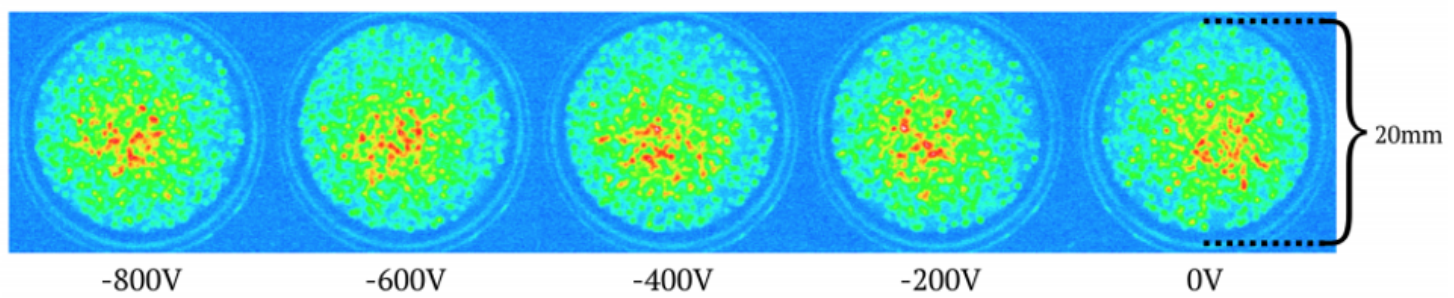

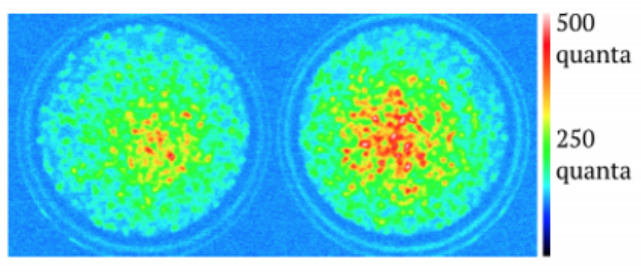

$+50 \mathrm{~V}$
$+100 \mathrm{~V}$

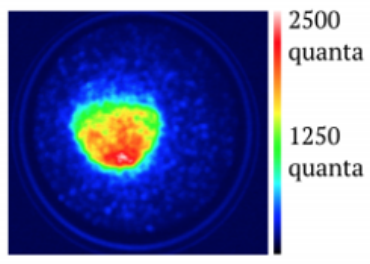

$+200 \mathrm{~V}$

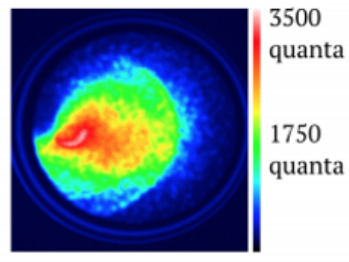

OV

$\mathrm{e}^{+}$implanted on MCP

Figure 8. Images obtained from charged particles impinging on an MCP set-up as a function of the MCP surface potential applied, to determine the secondary electron energies. Positron implantation energy set to 3.3 $\mathrm{keV}$ (target set to transmission mode). The last image shows the particle spot without the target present (only positrons transmitted). Image also from Ref. [30].

\section{Outlook and Conclusions}

The AEgIS experiment at the Antiproton Decelerator at CERN, has the objective to study the free fall of antimatter in Earth's gravitational field, using a pulsed cold antihydrogen-based atomic beam. Both the duration of the free fall and the vertical displacement of the horizontally travelling atoms will be measured, allowing a first test of the WEP with antimatter.

Several relevant results has recently been obtained towards the precise measurement of antimatter gravity with antihydrogen by the AEgIS collaboration:

- An optmized compression method for the antiprotons have been proposed to obtain a high catching efficiency [32].

- Positronium has been excited to the $n=3$ state, the first step in the two-step process to Rydberg excited Ps [28]. This is vital for the charge exchange process.

Alternative methods to produce Ps are still in development. A carbon-supported Ps transmission target was also tested to determine the feasibility for use within the main experiment. Another technique being explored is a self-supporting mesoporous film, currently in the prototype phase [33], to produce a high yield of Ps yield with low emission energies. A self-supporting target is designed with the intention of removing the emission of secondary charged particles $\left(e^{-}\right.$and $\left.e^{+}\right)$from being emitted with the Ps. Further tests are planned in the near future. 


\section{Acknowledgements}

This work was supported by the European Research Council, the European Social Fund, the Istituto Nazionale di Fisica Nucleare, the Austrian Ministry for Science, Research and Economy, the German Research Foundation the Research Council of Norway, the Bergen Research Foundation, the Russian Ministry of Education and Science, the Russian Academy of Sciences and the John Templeton Foundation and a Marie Sklodowska-Curie Innovative Training Network Fellowship of the European Commission's Horizon 2020 Programme under contract number 721559 AVA.

\section{References}

[1] J.Y. Hémery, S. Maury, Nucl. Phys. A 655, 354c (1999), doi:10.1016/S0375-9474(99)00223-7

[2] W. Pauli, in Niels Bohr and the Development of Physics, edited by W. Pauli, L. Rosenfeld, V. Weisskopt, (Pergamon Press, New York, 1999), p. 30

[3] D. S. Hajdukovic, Astrophys. Space Sci. 330, 1 (2010), doi:10.1007/s10509-010-0387-x

[4] A. P. Pullman, D. L. Lee, Phys. Rev. D 8, 2 (1973), doi:10.1103/PhysRevD.8.1226

[5] S. Schlamminger, K. -Y. Choi, T. A. Wagner, J. H. Gundlach, E. G. Adelberger, Phys. Rev. Lett. 100, 041101 (2008), doi:10.1103/PhysRevLett.100.041101

[6] R. S. Van Dyck Jr., D. J. Wineland, P. A. Ekstrom, H. G. Dehmelt, Appl. Phys. Lett. 28, 446 (1976), doi: http://dx.doi.org/10.1063/1.88793

[7] T. W. Darling, F. Rossi, G. I. Opat, G. F. Moorhead, Rev. Mod. Phys. 64, 1 (1992), doi:10.1103/RevModPhyys.64.237

[8] M. Amoretti et al (ATHENA Collaboration), Nature 419, 456 (2002), doi:10.1038/nature01096

[9] The ALPHA Collaboration, A. E. Charman, Nat. Commun. 4, 1785 (2013), doi:10.1038/ncomms2787

[10] A. Kellerbauer et al. (AEgIS Proto-Collaboration), Nucl. Instrum. Methods B 266, 351 (2008), doi:10.1016/j.nimb.2007.12.010

[11] G. Drobychev et al. (AEgIS Proto-Collaboration), Proposal to the SPS Experiments Committee SPSC-P-334, CERN, Geneva (2007), http://cds.cern.ch/record/1037532

[12] M. Deutch, Phys. Rev. 82, 455 (1951)

[13] B. I. Deutch et al., Proc. 1st Workshop on Antimatter Physics at Low Energies http://lss.fnal.gov/conf/C860410/ (1986)

[14] E. A. Hessels, D. M. Homan, M. J. Cavagnero, Phys. Rev. A. 57, 1669 (1998)

[15] M. Charlton, Phys. Lett. A 20, 143 (1990), doi:10.1016/0375-9601(90)90665-B

[16] T. Breeden, H. Metcalf, Phys. Rev. Lett. 47, 24 (1981), doi:10.1103/PhysRevLett.47.1726

[17] S. L. Rolston and G. Gabrielse, Hyperfine Interact. 44, 233 (1988)

[18] C. M. Surko, M. Leventhal, A. Passner, Phys. Rev. Lett. 62, 901 (1989), doi:10.1103/PhysRevLett.62.901

[19] S. Mariazzi, P. Bettotti, S. Larcheri, L. Toniutti, R. S. Brusa, Phys. Rev. B 81, 235418 (2010), doi:10.1103/PhysRevB.81.235418

[20] G. Consolati, R. Ferragut, A. Galarneau, F. Di Renzo, F. Quasso, Chem. Soc. Rev. 42, 3821 (2013), doi:10.1039/c2cs35454c

[21] S. Aghion et al (AEgIS Collaboration), Nucl. Instrum. Methods B 362, 86 (2015), doi:10.1016/j.nimb.2015.08.097

[22] S. Cialdi, I. Boscolo, F. Castelli, F. Villa, G. Ferrari, M. G. Giammarchi, Nucl. Instrum. Methods B 269, 1527 (2011), doi:10.1016/j.nimb.2011.04.108 
[23] M. K. Oberthaler et al (AEgIS Collaboration), Phys. Rev. A 54, 4 (1996), doi:10.1103/PhysRevA.54.3165

[24] G. Testera et al (AEgIS Collaboration), AIP Conf. Proc. 1037, 5 (2008), doi:10.1063/1.2977857

[25] S. Aghion et al (AEgIS Collaboration), Nature Commun. 5, 4538 (2014), doi: $10.1038 /$ ncomms5538

[26] C. Pistillo et al (AEgIS Collaboration), Hyperfine Interact. 233, 29 (2015), doi:10.1007/s10751015-1175-3

[27] S. L. Anderson et al, J. Phys. B 48, 204003 (2015), doi:10.1088/0953-4075/48/20/204003

[28] S. Aghion et al (AEgIS Collaboration), Phys. Rev. A 94, 012507 (2016), doi:10.1103/PhysRevA.94.012507

[29] D. B. Cassidy, S. H. M. Deng, H. K. M. Tanaka, A. P. Mills, Appl. Phys. Lett. 88, 194105 (2006), doi:10.1063/1.2203336

[30] S. Aghion et al. (AEgIS Collaboration), Nucl. Instrum. Methods B 407, 55 (2017), doi:10.1016/j.nimb.2017.05.059

[31] S. L. Andersen et al, Eur. Phys. J. D 68, 124 (2014), doi:10.1140/epjd/e2014-40762-x

[32] S. Aghion et al. (AEgIS Collaboration), Eur. Phys. J. D (2017) (Submitted)

[33] C. Evans et al. (in preparation) 